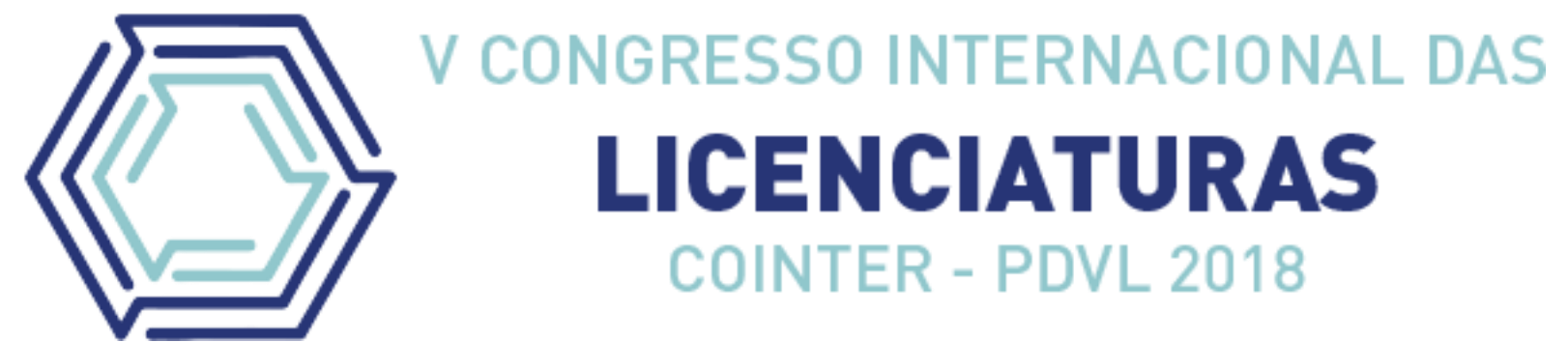

\title{
O USO DE OFICINAS TEMÁTICAS COMO FERRAMENTA AUXILIADORA NO ENSINO DE QUÍMICA
}

\author{
Apresentação: Comunicação Oral \\ Maria José da Silva Lima ${ }^{1}$; Palloma Joyce de Aguiar Silva ${ }^{2}$; Josefa Luana da Silva Sousa ${ }^{3}$; \\ Kymberli Francisca de Souza ${ }^{4}$; Sanderson Hudson da Silva Malta ${ }^{5}$ \\ DOI: https://doi.org/10.31692/2358-9728.VCOINTERPDVL.2018.00123
}

\section{Resumo}

Este trabalho apresenta a oficina temática como metodologia em química capaz de ser instrumento facilitador no processo de ensino-aprendizagem. Para isso, fez-se necessário a produção da oficina intitulada "café com Química" para análise dos pressupostos dessa metodologia. Esse trabalho é resultado de um estudo que conecta estudantes licenciandos em Química do Instituto Federal de Pernambuco e alunos do ensino médio de uma escola da rede pública estadual de Referência da cidade de Barra de Guabiraba/ PE. Trata-se de uma intervenção interdisciplinar que utiliza as oficinas como metodologia de ensino. Teve como objetivo, analisar o efetivo processo de aprendizagem numa perspectiva interdisciplinar através da oficina temática como metodologia de ensino em química, correlacionando os saberes. Resulta da análise de uma atividade de caráter intervencionista que culminou da apresentação da oficina denominada "café com química". A oficina foi apresentada durante um evento interno da própria escola onde a ideia principal era fazer a ponte entre os conhecimentos científicos e os adquiridos fora da sala de aula; no dia-a-dia. A metodologia utilizada seguiu numa abordagem qualitativa e de cunho descritivo. Os procedimentos adotados priorizaram: analise das observações do alunado, utilização de objetos tecnológicos para auxilio de aula expositiva e utilização de materiais produzidos pelos alunos intervencionistas. Seguindo os três momentos pedagógicos: problematização inicial, organização do conhecimento e aplicação do conhecimento. $\mathrm{O}$ estudo está embasado em pesquisas que tem como fundamentação a aprendizagem significativa, o ensino das ciências e a interdisciplinaridade. A finalidade proposta neste trabalho é de proporcionar a integração do currículo escolar com os conteúdos históricos que são aprendidos no dia a dia, e introduzir metodologias de ensino que sejam

\footnotetext{
1 Licenciatura em Química, Instituto Federal de Educação, Ciência e Tecnologia de Pernambuco, srmarialima@gmail.com

2 Licenciatura em Química, Instituto Federal de Educação, Ciência e Tecnologia de Pernambuco, palloma_joyce_aguiar@hotmail.com

3 Licenciatura em Química, Instituto Federal de Educação, Ciência e Tecnologia de Pernambuco, lu.souz@hotmail.com

4 Licenciatura em Química, Instituto Federal de Educação, Ciência e Tecnologia de Pernambuco, kymberlisouza@hotmail.com

5 Especialista, Instituto Federal de Educação, Ciência e Tecnologia de Pernambuco, sanderson.malta@vitoria.ifpe.edu.br
} 
capazes de quebrar os paradigmas existentes no ensino das ciências. Foi observado que os alunos consideram mais interessante uma aula que faça uma ponte entre os conhecimentos curriculares e sua realidade, correlacionando com outras áreas do conhecimento, facilitando assim a compreensão dos conteúdos vistos em sala.

Palavras-Chave: Ensino de Ciências. Metodologias de Ensino, Oficinas temáticas.

\begin{abstract}
This work presents the thematic workshop as methodology in chemistry capable of being an instrument facilitator in the teaching-learning process. For this, it was necessary to produce the workshop entitled "coffee with chemistry" to analyze the assumptions of this methodology. This work is the result of a study that connects undergraduate students in Chemistry of the Federal Institute of Pernambuco and high school students of a public school in the city of Barra de Guabiraba / PE. It is an interdisciplinary intervention that uses the workshops as teaching methodology. It aimed to analyze the effective learning process in an interdisciplinary perspective through the thematic workshop as methodology of teaching in chemistry, correlating the knowledge. It results from the analysis of an interventionist activity that culminated in the presentation of the workshop called "coffee with chemistry". The workshop was presented during an internal school event where the main idea was to bridge the gap between scientific and acquired knowledge outside the classroom; day to day. The methodology used followed a qualitative and descriptive approach. The procedures adopted prioritized: analysis of students' observations, use of technological objects to help with expository class and use of materials produced by interventionist students. Following the three pedagogical moments: initial problematization, organization of knowledge and application of knowledge. The study is based on research that is based on meaningful learning, teaching science and interdisciplinarity. The purpose of this work is to provide the integration of the school curriculum with the historical contents that are learned from day to day, and to introduce teaching methodologies that are capable of breaking the existing paradigms in science teaching. It was observed that the students consider more interesting a class that bridges the curricular knowledge and its reality, correlating with other areas of knowledge, thus facilitating the understanding of the contents seen in the classroom.
\end{abstract}

Keywords : science teaching, teaching methodologies, thematic offices.

\title{
Introdução
}

Um dos maiores desafios que está presente no ensino de química é a busca por diferentes metodologias de ensino que permita com que o estudante se interesse por esta área do saber e encontre nela uma correlação com o dia a dia. É notório que essa disciplina se torna desinteressante para os estudantes quando ministrada de maneira que não possibilite correlação com o cotidiano.

Os parâmetros curriculares nacionais para o ensino médio discorrem sobre a importância do currículo escolar para a formação efetiva e total do ser humano, não só no que 
diz respeito a sua vivência em sociedade, mas também para auto formação. No que diz respeito ao ensino de ciências, o currículo necessita está organizado de maneira que possibilite no momento do processo de ensino o desenvolvimento de competências e habilidades necessárias para o entendimento da mesma como fruto de produções humanas, entendendo seu desenvolvimento e seus vieses.

Analisando esses fatos é possível perceber que a química no ensino médio, deve possibilitar aos alunos não só o contato com os conteúdos pré-programados, não só saber identificar os processos que ocorrem durante uma experimentação ou um exemplo dado em sala de aula pelo professor, mas também saber identificar todas as articulações e aplicações da ciência na comunidade. Sendo assim, os conteúdos devem ser abordados de forma que possibilitem a contextualização do conhecimento, permitindo a formação de uma aprendizagem significativa.

Tendo em vista a grande importância que os conhecimentos sobre as ciências têm para uma participação mais ativa dos cidadãos na sociedade é que se apresentam metodologias de ensino que procuram tratar o conhecimento de maneira interligada e contextualizada, envolvendo também os alunos no processo ativo de construção do conhecimento.

As oficinas temáticas estão sujeitas a diferentes interpretações, mas apresenta um foco central dependendo de qual seu intuito. Apresentam características próprias e pode ser utilizada para diferentes finalidades, no que diz respeito ao ensino, as oficinas podem ser utilizadas para aproximar os conteúdos disciplinares às vivências do dia a dia dos alunos, como forma de organizar os conhecimentos e promover a aprendizagem.

Podem também ser utilizadas como forma de contextualizar o conhecimento e possibilita a abordagem interdisciplinar dos conteúdos. A contextualização pode ser inserida no currículo educacional através de temas que envolvam a sociedade e situações que não fujam da realidade dos educandos e que possibilite de forma integrada a discussão dos conteúdos correlacionando-os entre si. Possibilita a participação ativa dos alunos na formação do conhecimento.

As oficinas apresentam cunho experimental e devem ser planejadas de modo que provoque a curiosidade e levante questionamentos sobre os conceitos abordados e as aplicações na sociedade. A maneira que o conteúdo é tratado é que será alicerce para a construção interdisciplinar dos conteúdos químicos e o desenvolvimento de atitudes cidadãs. A interdisciplinaridade tem o papel de auxiliar na construção de novos conhecimentos que estejam 
associados a partir daqueles conhecimentos que já haviam sido fragmentados e muitas vezes não definiam um significado exato por não ser relevante no cotidiano. Este trabalho apresenta uma experiência de ensino através de oficinas temáticas que procura a aprendizagem científica e o estabelecimento de relações com a sociedade.

\section{Fundamentação Teórica}

A ideia de contextualização surge no Brasil em 1996 com a implementação da Lei de Diretrizes e Bases da Educação (LDB 9.394/96) que tem o papel de definir e regularizar a educação Brasileira. O PCN do ensino médio (1999) discorre sobre a necessidade de dar significado ao conhecimento escolar, mediante a contextualização; evitar a compartimentalização, mediante a interdisciplinaridade; e incentivar o raciocínio e a capacidade de aprender".

“Os conteúdos curriculares no ensino médio, na grande maioria das escolas, são organizados de maneira fracionada em disciplinas, e desenvolvidos de forma fragmentada, dificultando a compreensão do conhecimento como um todo integrado" (GALLO, 1997; MORIN, 2000 et al.). Pensando na correlação das disciplinas para efetivação do conhecimento e da formação cidadã como previsto em primeiro momento nos Parâmetros Curriculares Nacionais é que se faz necessário discutir metodologias de ensino que possibilitem atingir a finalidade educacional de maneira democrática, e que tenham o papel de correlacionar o ensino de química com a realidade social dos alunos, tornando os mesmos sujeitos críticos e capazes de tomar decisões em meio a sua realidade.

A associação dos conhecimentos científicos com objetos do cotidiano faz com que os alunos assimilem com mais facilidade pois poderão associa-los a momentos práticos fugindo do abstrato e da irrealidade. "A contextualização deve ser vista como um dos instrumentos para a concretização da ideia de interdisciplinaridade e para favorecer a atribuição de significados pelo aluno no processo de ensino e aprendizagem" (BRASIL, 2006, p. 95).

Segundo Almeida (2008), um dos objetivos da química é que o jovem reconheça o valor da ciência na busca do conhecimento da realidade objetiva e os insira no cotidiano. É necessário que os estudantes consigam identificar a importância da ciência em sua realidade e os avanços que a sociedade obteve a partir da mesma.

Zanon e Palharine (1995) enfatizam que os conteúdos são contextualizados de forma correta diminui a distância e a dificuldade, despertando o interesse e a motivação dos alunos. 
As oficinas temáticas são manifestações necessárias para a inter-relação da ciência com a realidade do aluno, facilitado assim o processo de ensino. Trata-se, sim, de abordar dados, informações e conceitos para que se possa conhecer a realidade, avaliar situações e soluções e propor formas de intervenção na sociedade (MARCONDES et al., 2007).

Segundo Hodson (1994), as oficinas caracterizam-se também pela utilização de instrumentos experimentais, que na maioria das vezes são realizadas pelos alunos, despertando assim a curiosidade e o interesse pelos fenômenos científicos.

\section{Metodologia}

A oficina tratada neste trabalho foi estruturada segundo os três momentos pedagógicos (Delizoicov; Angotti, 1990). Foi realizada em parceria entre estudantes da Licenciatura em Química do Instituto Federal de Pernambuco/ CVSA e alunos do $2^{\circ}$ ano do ensino médio de uma escola pública de referência da cidade de Barra de Guabiraba/ PE e professores da escola supracitada.

A oficina caracteriza-se por um projeto de intervenção pedagógica que contou com a presença de 25 participantes e foi realizada na própria escola durante o evento interno, nomeado "II fórum do ensino médio", evento este que ofertava minicursos, oficinas e palestras e estava voltado para a educação integral e a formação do aluno enquanto cidadão. A oficina objetivava a análise do efetivo processo de aprendizagem em uma abordagem interdisciplinar dos conteúdos com a utilização de oficinas temáticas. Essa oficina foi desenvolvida em três momentos pedagógicos listados a seguir:

$1^{\mathrm{o}}$ momento pedagógico - problematização inicial: Nesse momento, os alunos foram questionados a critério de análise sobre o que eles sabiam dos processos químicos por trás do café que chega as nossas casas. A ideia principal desse momento era saber os conhecimentos prévios dos alunos sobre o assunto.

Questionário $\left(1^{\circ}\right.$ momento $)$

Você sabe quais os processos químicos por trás do café que chega a nossas casas?

\section{Quadro 1 Fonte: próprio autor}

Logo em seguida, foi apresentado aos alunos por meio de vídeos e pesquisas de bases científicas sobre a historicidade do café e todos os momentos históricos que tiveram essa semente como protagonista no Brasil. 
$2^{\circ}$ momento pedagógico - organização do conhecimento: Nesse momento, foram abordados alguns tópicos necessários para o entendimento da composição química do café. Os nutrientes, as funções orgânicas, os efeitos do café no organismo, os métodos de conservação foram informações expostas através de vídeos.

No $3^{\mathrm{o}}$ momento pedagógico ocorreu a aplicação do conhecimento onde os alunos puderam produzir manualmente o pó do café a partir de diferentes moagens com grão já submetidos a torra anteriormente. Os alunos puderam também nesse momento conhecer os métodos de análise do café (sensorial e química) e os processos químicos que acompanham o café desde a colheita até a sua distribuição. Alguns instrumentos são indispensáveis nesse momento como: moedores, o próprio grão de café e medidores de acidez e coadores. A preparação do café foi toda feita por parte dos alunos com supervisão dos licenciandos. Enquanto estava sendo preparado foram senso sanadas algumas dúvidas que foram levantadas durante os processos.

No último momento ocorreu um pequeno debate com os alunos para analisar se a oficina tinha contribuído para a associação dos conceitos científicos e construção do conhecimento.

\section{Resultados e Discussão}

As informações da oficina foram analisadas a partir das descrições e participação à luz da quarta geração da avaliação de Guba e Lincoln (1988), que trata da avaliação como um objeto integrado no processo de ensino a partir de instrumentos predominantemente qualitativos.

No momento inicial quando questionados sobre os processos químicos por trás do café que chega a nossas casas percebeu-se que os alunos não conseguiam fundamentar suas ideias. Alguns alunos conseguiam identificar que o café afeta o corpo humano por causa de seus componentes e que tem o poder de deixar o ser humano ativo por mais tempo, mas não conseguiam explicar porque isso acontecia.

Ao analisarmos as respostas, após o desenvolvimento da oficina temática, constatamos que os alunos conseguiram explicar o processo de produção de café desde sua colheita até a produção da bebida utilizando conceitos científicos e relacionando esses conceitos com práticas no dia a dia, como por exemplo a análise sensorial, permitindo com que os alunos conseguissem identificar os fatores que permitem as alterações tanto no aroma quanto no sabor da segunda bebida mais consumida no mundo. 
O aluno A que no momento inicial afirmou apenas que sabia que havia vários compostos químicos no grão de café, na finalização da oficina conseguiu elencar alguns pontos importantes que foram expostos: "Foi interessante! Aprendi que os compostos podem ser identificados em um laboratório equipado, mas que eles se manifestam em nosso corpo através das sensações. Por isso me sinto tão bem quando tomo um cafezinho".

O aluno B conseguiu exemplificar na sua fala a influência que o tipo de torra e moagem tem sobre o produto final: "É importante que a torra e a moagem sejam feitas de maneira correta porque isso também influência na bebida. Tem gente que gosta de um sabor mais forte e outros um sabor mais fraco, por isso é bom fazer sempre uma análise”.

A partir da observação das falas dos alunos percebeu-se que as oficinas temáticas são instrumentos facilitadores no ensino tendo em vista que permitem a construção do conhecimento científico, a contextualização e interligação com outras áreas do saber. Notou-se que os alunos ficam mais motivados com metodologias de ensino que permitam sua participação ativa e que não os deixe como meros espectadores.

\section{CONCLUSÃO}

Percebe-se que os assuntos de química quando tratados de forma interdisciplinar e contextualizada permite que os alunos se sintam motivados e interessados pelas aulas. As diferentes metodologias de ensino permitem que os alunos interajam e participem ativamente nas atividades facilitando a aprendizagem e desenvolvendo o senso-crítico. Percebeu-se que as oficinas temáticas apresentam um espaço capaz de correlacionar conceitos de forma a contribuir para a melhor compreensão do mundo. O ensino contextualizado é motivador do aprendizado.

As oficinas temáticas conseguem prender a atenção dos alunos, facilitando assim a articulação do raciocínio, utilizando-se de conceitos que são necessários para o saber curricular e outros já adquiridos no dia-a-dia. Os alunos mostraram satisfação em aprender os conteúdos químicos a partir de um elemento tão presente nas suas vidas.

Percebeu-se também que os estudantes conseguiram quebrar alguns paradigmas relacionados a disciplina de química quando associaram que a ciência faz parte de sua vida diária. A apropriação dos conceitos é um fato importante que foi observado após a realização da oficina e análise das falas durante o debate final. É importante salientar que dependendo de qual seja a intencionalidade da oficina se faz necessário uma correlação com outras áreas dos saberes no momento inicial, para que os alunos se sintam mais familiarizados com os conceitos. 


\section{Referências}

ALMEIDA, E. Contextualização no ensino de química: motivando alunos de ensino médio 2008.

BRASIL. Ministério da educação. Secretaria de ensino básico. Orientações curriculares para o ensino médio: ciências da natureza, matemática e suas tecnologias.

BRASIL (país) Secretaria de Educação Média e Tecnológica - Ministério da Educação e Cultura. Parâmetros Curriculares Nacionais - Ensino Médio: Bases Legais. Brasília: MEC/SEMTEC, vol. 1, 1999

DELIZOICOV, D.; ANGOTTI, J. A. Metodologia do ensino de ciências. São Paulo: Cortez, 1990

DELIZOICOV, D. Problemas e Problematizações. In: PIETROCOLA, M. (org.). Ensino de Física? Conteúdo, metodologia e epistemologia numa concepção integradora. Florianópolis: Ed. da UFSC, 2002.

DIRETRIZES E BASES DA EDUCAÇÃO NACIONAL. Lei nº 9.394 Bauru: São Paulo: Endipro 1996.

GALLO, S. Conhecimento, transversalidade e educação: para além da interdisciplinaridade. Impulso: Revista de Ciências Sociais e Humanas. Piracicaba: Ed. UNIMEP, v. 10, n. 21, 1997

HODSON, D. "Hacia un Enfoque Más Crítico del Trabajo de Laboratorio”. Enseñanza de Las Ciencias, 1994.

MARCONDES, M. E. R. et al. Oficinas Temáticas no Ensino Público visando a Formação Continuada de Professores. São Paulo: Imprensa Oficial do Estado de São Paulo, 2007.

SANTOS, W. L. P. Contextualização no ensino de ciências por meio de temas CTS em uma perspectiva crítica. Ciência \& Ensino, v.1, novembro de 2007.

ZANON, L.B. e PALHARINI, E. M. A química no ensino fundamental de ciências. Química nova na escola, $n^{\circ} .2$ pg. $15-18,1995$. 